\title{
REPRESENTATIONS OF EULER CLASSES
}

\author{
HOWARD OSBORN ${ }^{1}$
}

To Professor S. S. Chern on his sixtieth birthday

\begin{abstract}
For any endomorphism $K$ of an oriented module $F$ with inner product there is an element $\mathrm{pf} K$ in the ground ring $R$, a constant multiple of the classical pfaffian in the case $F=R^{2 n}$. If $R$ is the algebra of even-dimensional differential forms on a smooth manifold, and if $F$ is the tensor product of $R$ and the module of sections of an oriented $2 n$-plane bundle, then any connection in the bundle induces a curvature transformation $K: F \rightarrow F$ for which $(4 \pi)^{-n}$ pf $K$ represents the Euler class. Properties of Euler classes are immediate consequences of corresponding properties of pf.
\end{abstract}

1. Pfaffians. Let $R$ be any commutative ring with unit. An $R$-module $F$ is orientable if $\bigwedge^{m} F$ is free of rank 1 for some $m>0$; according to [4] there is at most one such integer $m$, and throughout this note we assume that $m$ is an even integer $2 n$. An orientable module $F$ is oriented by assigning a generator $S \in \bigwedge^{2 n} F$.

An inner product on $F$ is any nondegenerate symmetric bilinear map $\langle\rangle:, F \times F \rightarrow R$, not necessarily positive-definite in any sense. An inner product on $F$ induces inner products on $\Lambda^{p} F$ for $p=0, \cdots, 2 n$ in the usual fashion, and we assume $\langle S, S\rangle= \pm 1$ for the orientation $S$; the \pm sign depends on $\langle$,$\rangle . The Hodge operator *: \bigwedge^{n} F \rightarrow \bigwedge^{n} F$ in dimension $n$ is given by requiring

$$
\left\langle e_{1} \wedge \cdots \wedge e_{n}, *\left(f_{1} \wedge \cdots \wedge f_{n}\right)\right\rangle=\left\langle e_{1} \wedge \cdots \wedge e_{n} \wedge f_{1} \wedge \cdots \wedge f_{n}, S\right\rangle
$$

for any $e_{1}, \cdots, e_{n}, f_{1}, \cdots, f_{n} \in F$.

Definition 1.1. For any endomorphism $K$ of $F$ the pfaffian is the element pf $K=(-1)^{n(n-1) / 2} \operatorname{tr} * \circ \Lambda^{n} K$, where $*_{\circ} \bigwedge^{n} K$ is a composition of endomorphisms of $\Lambda^{n} F$ and "tr" means "trace".

Let ${ }^{t} K$ denote the transpose of $K:\left\langle e,{ }^{t} K f\right\rangle=\langle K e, f\rangle$.

Proposition 1.2. pf $K=\operatorname{pf}\left(-{ }^{t} K\right)$.

Received by the editors December 14, 1970 and, in revised form, March 19, 1971. AMS 1970 subject classifications. Primary 15A15, 57D20, 58A10; Secondary 53B20, 58A99.

Key words and phrases. Pfaffians, Hodge operator, connections, curvature transformations, Euler classes, Avez-Chern theorem, Gauss-Bonnet theorem.

${ }^{1}$ Research supported by National Science Foundation Grant GP-13145. 
Proof. One easily computes ${ }^{t} *=(-1)^{n} *$, and since ${ }^{t}\left(\Lambda^{n} K\right)=$ $\Lambda^{n}\left({ }^{t} K\right)$ it follows that $\operatorname{tr} * \circ \Lambda^{n} K=\operatorname{tr} \bigwedge^{n} K \circ *=\operatorname{tr}^{t}\left(\bigwedge^{n} K \circ *\right)=$ $\operatorname{tr}^{t} *^{t}{ }^{t}\left(\bigwedge^{n} K\right)=\operatorname{tr} * \circ \Lambda^{n}\left(-{ }^{t} K\right)$.

We shall need the following two properties of $*$.

Lemma 1.3. $\bigwedge^{n t} A \circ * \circ \bigwedge^{n} A=(\operatorname{det} A) *$ for any $A \in \operatorname{Hom}(F, F)$.

Proof.

$$
\begin{aligned}
\left\langle e_{1} \wedge \cdots \wedge e_{n},\left(\wedge^{n t} A \circ * \circ \wedge^{n}\right.\right. & \left.A) f_{1} \wedge \cdots \wedge f_{n}\right\rangle \\
& =\left\langle A e_{1} \wedge \cdots \wedge A e_{n}, *\left(A f_{1} \wedge \cdots \wedge A f_{n}\right)\right\rangle \\
& =\left\langle A e_{1} \wedge \cdots \wedge A e_{n} \wedge A f_{1} \wedge \cdots \wedge A f_{n}, S\right\rangle \\
& =(\operatorname{det} A)\left\langle e_{1} \wedge \cdots \wedge e_{n}, *\left(f_{1} \wedge \cdots \wedge f_{n}\right)\right\rangle .
\end{aligned}
$$

Lemma 1.4. For any invertible $A \in \operatorname{Hom}(F, F)$ let $\langle,\rangle^{\prime}$ be the inner product given by $\langle e, f\rangle^{\prime}=\langle A e, A f\rangle$; then the corresponding Hodge operator $*^{\prime}: \bigwedge^{n} F \rightarrow \Lambda^{n} F$ satisfies $*^{\prime}=\Lambda^{n} A^{-1} \circ * \circ \Lambda^{n} A$.

Proof. For $S^{\prime}=\left(\bigwedge^{2 n} A^{-1}\right) S$ one has

hence

$$
\left\langle S^{\prime}, S^{\prime}\right\rangle^{\prime}=\left\langle\left(\bigwedge^{2 n} A\right) S^{\prime},\left(\bigwedge^{2 n} A\right) S^{\prime}\right\rangle=\langle S, S\rangle= \pm 1 ;
$$

$$
\begin{aligned}
\left\langle e_{1} \wedge \cdots \wedge e_{n}, *^{\prime}\left(f_{1}\right.\right. & \left.\left.\wedge \wedge f_{n}\right)\right\rangle^{\prime} \\
& =\left\langle e_{1} \wedge \cdots \wedge e_{n} \wedge f_{1} \wedge \cdots \wedge f_{n}, S^{\prime}\right\rangle^{\prime} \\
& =\left\langle A e_{1} \wedge \cdots \wedge A e_{n} \wedge A f_{1} \wedge \cdots \wedge A f_{n}, S\right\rangle \\
& =\left\langle A e_{1} \wedge \cdots \wedge A e_{n}, *\left(A f_{1} \wedge \cdots \wedge A f_{n}\right)\right\rangle \\
& =\left\langle e_{1} \wedge \cdots \wedge e_{n},\left(\wedge^{n} A^{-1} \circ * \circ \wedge^{n} A\right) f_{1} \wedge \cdots \wedge f_{n}\right\rangle^{\prime} .
\end{aligned}
$$

Proposition 1.5. $\operatorname{pf}\left(A \circ K^{\circ} A\right)=(\operatorname{det} A) \operatorname{pf} K$.

Proof. Lemma 1.3 gives

$$
\begin{aligned}
\operatorname{tr}\left(* \circ \Lambda^{n}\left(A \circ K \circ{ }^{t} A\right)\right) & =\operatorname{tr}\left(* \circ \Lambda^{n} A \circ \Lambda^{n} K \circ \Lambda^{n t} A\right) \\
& =\operatorname{tr}\left(\bigwedge^{n} t A \circ * \circ \Lambda^{n} A \circ \Lambda^{n} K\right) \\
& =(\operatorname{det} A) \operatorname{tr}\left(* \circ \Lambda^{n} K\right) .
\end{aligned}
$$

Corollary 1.6. If $K=A \circ J \circ{ }^{t} A$ then $(\operatorname{pf} K)^{2}(\operatorname{det} J)=(\operatorname{pf} J)^{2}(\operatorname{det} K)$.

Proof. Both sides are equal to $(\operatorname{det} A)^{2}(\operatorname{pf} J)^{2}(\operatorname{det} J)$.

If $E$ and $F$ are oriented inner product spaces of dimensions $2 p$ and $2 q$ then the direct sum $E \oplus F$ is also an oriented inner product space with respect to the obvious definitions. Let $*$ " be the resulting Hodge operator on $\bigwedge^{p+q}(E \oplus F)$, this module being canonically isomorphic to the direct sum $\sum_{r+s=p+q} \Lambda^{r} E \otimes \Lambda^{s} F$. If $\iota$ and $\rho$ are the injection and projection for 
the summand $\Lambda^{p} E \otimes \Lambda^{q} F$ then for the Hodge operators $*$ and $*^{\prime}$ on $\Lambda^{p} E$ and $\Lambda^{q} F$ one easily verifies $\rho \circ *^{\prime \prime} \circ \iota=(-1)^{p q} * \otimes *^{\prime}$.

Proposition 1.7. For any $J: E \rightarrow E$ and $K: F \rightarrow F$ it follows that $\operatorname{pf}(J \oplus K)=(\operatorname{pf} J)(\operatorname{pf} K)$.

Proof. $*^{\prime \prime} \circ \Lambda^{p+q}(J \oplus K)$ interchanges the summands $\Lambda^{r} E \otimes \Lambda^{8} F$ and $\bigwedge^{2 p-r} E \otimes \Lambda^{2 q-s} F$ of $\bigwedge^{p+q}(E \oplus F)$, so that only $\bigwedge^{p} E \otimes \Lambda^{q} F$ plays a role in commuting the trace. Hence

$$
\begin{aligned}
\operatorname{tr}\left(*^{\prime \prime} \circ \bigwedge^{p+q}(J \oplus K)\right) & =\operatorname{tr}\left(\rho \circ *^{\prime \prime} \circ \bigwedge^{p+q}(J \oplus K) \circ \iota\right) \\
& =\operatorname{tr}\left(\left(\rho \circ *^{\prime \prime} \circ \iota\right) \circ\left(\rho \circ \bigwedge^{p+q}(J \oplus K) \circ \iota\right)\right) \\
& =(-1)^{p q} \operatorname{tr}\left(\left(* \otimes *^{\prime}\right) \circ\left(\bigwedge^{p} J \otimes \bigwedge^{q} K\right)\right) \\
& =(-1)^{p q} \operatorname{tr}\left(\left(* \circ \bigwedge^{p} J\right) \otimes\left(*^{\prime} \circ \bigwedge^{q} K\right)\right) \\
& =(-1)^{p q}\left(\operatorname{tr} * \bigwedge^{p} J\right)\left(\operatorname{tr} *^{\prime} \circ \bigwedge^{q} K\right),
\end{aligned}
$$

and one inserts the \pm sign of Definition 1.1 to obtain the result.

A free module $F$ of rank $2 n$ is oriented by ordering a basis $e_{1}, \cdots, e_{2 n}$ up to even permutation, and the usual inner product is given by $\left\langle\boldsymbol{e}_{i}, \boldsymbol{e}_{j}\right\rangle=$ $\delta_{i j}$. One easily checks for any permutation $\pi$ of $(1, \cdots, 2 n)$ and $S=$ $e_{1} \wedge \cdots \wedge e_{2 n}$ that $*\left(e_{\pi 1} \wedge \cdots \wedge e_{\pi n}\right)=(-1)^{n} \varepsilon_{\pi} e_{\pi(n+1)} \wedge \cdots \wedge e_{\pi(2 n)}$, where $\varepsilon_{\pi}$ is the parity of $\pi$; this implies

$$
*\left(e_{\pi 1} \wedge e_{\pi 3} \wedge \cdots \wedge e_{\pi(2 n-1)}\right)=(-1)^{n(n+1) / 2} \varepsilon_{\pi} e_{\pi 2} \wedge e_{\pi 4} \wedge \cdots \wedge e_{\pi(2 n)} .
$$

Proposition 1.8. If $\left(K_{j}^{i}\right)$ represents an endomorphism $K$ of a free $R-$ module $F$ with respect to the basis $e_{1}, \cdots, e_{2 n}$, then

$$
\text { pf } K=(-1)^{n} \sum_{\Pi} \varepsilon_{\pi} K_{\pi 2}^{\pi 1} \cdots K_{\pi(2 n)}^{\pi(2 n-1)}
$$

where $\Pi$ is the set of those permutations $\pi$ of $(1, \cdots, 2 n)$ with $\pi 2<\cdots<$ $\pi(2 n)$.

Proof. Let $P \subset \Pi$ be the set of permutations $\pi$ satisfying both $\pi 1<\cdots<\pi(2 n-1)$ and $\pi 2<\cdots<\pi(2 n)$, for which

$$
\left\{e_{\pi 2} \wedge \cdots \wedge e_{\pi(2 n)} \mid \pi \in P\right\}
$$

is a basis of $\bigwedge^{n} F$, and let $T$ be the set of all permutations of $(1, \cdots, n)$. Then

$$
\begin{aligned}
\left(\bigwedge^{n} K\right)\left(e_{\pi 2} \wedge\right. & \left.\cdots \wedge e_{\pi(2 n)}\right)=\sum_{i_{1}} \cdots, i_{n} \\
& \left(K_{\pi 2}^{i_{1}} \cdots K_{\pi(2 n)}^{i_{n}}\right) e_{i_{1}} \wedge \cdots \wedge e_{i_{n}} \\
& =\left\{\sum_{T} \varepsilon_{r} K_{\pi 2}^{\pi(2 \tau(1)-1)} \cdots K_{\pi(2 n)}^{\pi(2 \tau(n)-1)}\right\} e_{\pi 1} \wedge \cdots \wedge e_{\pi(2 n-1)}+\cdots,
\end{aligned}
$$

where " $+\cdots$ " represents summands $e_{i_{1}} \wedge \cdots \wedge e_{i_{n}}$ with

$$
\left\{i_{1}, \cdots, i_{n}\right\} \neq\{\pi 1, \cdots, \pi(2 n-1)\} .
$$


Hence

$$
\begin{aligned}
(* & \left.\circ{ }^{n} K\right)\left(e_{\pi 2} \wedge \cdots \wedge e_{\pi(2 n)}\right) \\
& =\left\{(-1)^{n(n+1) / 2} \varepsilon_{\pi} \sum_{T} \varepsilon_{\tau} K_{\pi 2}^{\pi(2 \tau(1)-1)} \cdots K_{\pi(2 n)}^{\pi(2 \tau(n)-1)}\right\} e_{\pi 2} \wedge \cdots \wedge e_{\pi(2 n)}+\cdots,
\end{aligned}
$$

where " $+\cdots$ " now represents summands $e_{i_{1}} \wedge \cdots \wedge e_{i_{n}}$ with

Hence

$$
\left\{i_{1}, \cdots, i_{n}\right\} \neq\{\pi 2, \cdots, \pi(2 n)\} \text {. }
$$

$$
\begin{aligned}
\operatorname{tr} * \circ \bigwedge^{n} K & =(-1)^{n(n+1) / 2} \sum_{P, T} \varepsilon_{\pi} \varepsilon_{\tau} K_{\pi 2}^{\pi(2 r(1)-1)} \cdots K_{\pi(2 n)}^{\pi(2(2(n)-1))} \\
& =(-1)^{n(n+1) / 2} \sum_{\Pi} \varepsilon_{\pi} K_{\pi 2}^{\pi 1} \cdots K_{\pi(2 n)}^{\pi(2 n-1)} .
\end{aligned}
$$

We remark that except for a constant factor $\pm 2^{n}$ Proposition 1.8 states that pf $K$ is the classical pfaffian of $\left(K_{j}^{i}\right)$, usually defined only when $\left(K_{j}^{i}\right)$ is skew-symmetric. If $R$ contains $\frac{1}{2}$ then Proposition 1.8 implies that pf $K=$ pf $\frac{1}{2}\left(K-{ }^{t} K\right)$, so that pf $K$ depends only on the skew-symmetric part of $K$. For certain ground rings $R$, including the one considered in the next section, a localization procedure can be applied to obtain the same result for any oriented $R$-module $F$ with inner product.

Assume once more that $F$ is free of rank $2 n$, and let $J: F \rightarrow F$ have matrix representation consisting of blocks $\left(\begin{array}{cc}0 & -1 \\ 1 & 0\end{array}\right)$ down the main diagonal; then $\operatorname{det} J=1$, and Proposition 1.7 implies $\mathrm{pf} J= \pm 2^{n}$. Since any skewsymmetric matrix $(K)$ is of the form $\left(A \circ J^{\circ} A\right)$ for some endomorphism $A$, Corollary 1.6 becomes $(\mathrm{pf} K)^{2}=2^{2 n} \operatorname{det} K$. As in the preceding paragraph this result extends to the skew-symmetric endomorphisms $K$ of any oriented module $F$ with inner product over certain ground rings $R$.

2. Euler classes. For any smooth manifold $M$ let $\mathscr{E}$ be the $C^{\infty}(M)$ module of differentials on $M$, i.e., the smooth sections of the cotangent bundle, let $\left(\Lambda^{*} \mathscr{E}, d\right)$ and $H^{*}(M)$ be the de Rham complex and de Rham cohomology of $M$, and let $R$ be the even-dimensional subring ${\Lambda^{2 *} \mathscr{E} \subset}^{2}$ $\Lambda^{*} \mathscr{E}$. If $\mathscr{F}$ is the $C^{\infty}(M)$-module of smooth sections of any oriented $2 n$-plane bundle $\xi$ over $M$, then any connection in $\xi$ may be regarded as a real linear map $D: \Lambda^{*} \mathscr{E} \otimes \mathscr{F} \rightarrow \Lambda^{*} \mathscr{E} \otimes \mathscr{F}$ for which the curvature is a $\Lambda^{*} \mathscr{E}$-module endomorphism $D \circ D$ of degree 2 ; it follows that the curvature induces an $R$-linear endomorphism $K: F \rightarrow F$ for $F=R \otimes \mathscr{F}$. The orientation of $\xi$ induces an orientation of $F$, and any riemannian metric on $\xi$ induces an inner product on $F$.

In case $\left(K_{j}^{i}\right)$ represents the endomorphism induced by $K$ for some trivialization of $\xi$ over an open $U \subset M$, then the Euler class $e(\xi) \in H^{2 n}(M)$ is represented by a closed $2 n$-form whose restriction to $U$ is $(-4 \pi)^{-n} \sum_{\Pi} \varepsilon_{\pi} K_{\pi 2}^{\pi 1} \cdots K_{\pi(2 n)}^{\pi(2 n-1)}$. (See [5, e.g.].) It follows from Proposition 
1.8 that $e(\xi)$ is globally represented by $(4 \pi)^{-n}$ pf $K$. In fact one can easily develop geometric Euler classes ab initio in terms of the properties of $\mathrm{pf}$, as established in the preceding section, without reference to the trivializations of $\xi$; we sketch such a development.

The inner product on $F$ induces a corresponding bilinear form $\langle$, for the 2-sided $\Lambda^{*} \mathscr{E}$-module $\Lambda^{*} \mathscr{E} \otimes \mathscr{F}$, and a connection $D$ is metric whenever $\langle D e, f\rangle+\langle e, D f\rangle=d\langle e, f\rangle$. To verify that a given $D$ is metric it suffices to suppose that $e, f$ are of the forms $1 \otimes s, 1 \otimes t$ for sections $s, t$ of $\xi$; for convenience we write $s, t$ in place of $1 \otimes s, 1 \otimes t$.

LemMa 2.1. For any invertible endomorphism $A$ of $F$ let $\langle,\rangle^{\prime}$ be the inner product given by $\langle e, f\rangle^{\prime}=\langle A e, A f\rangle$, and extend $A$ to an endomorphism of $\Lambda^{*} \mathscr{E} \otimes \mathscr{F}$. Then if $D$ is a metric connection with respect to $\langle$,$\rangle it$ follows that $A^{-1} \circ D \circ A$ is a metric connection with respect to $\langle,\rangle^{\prime}$; furthermore the curvature $K^{\prime}$ of $D^{\prime}$ is given by $K^{\prime}=A^{-1} \circ K \circ A$.

Proof. For $\theta \in \bigwedge^{p} \mathscr{E}$ and $s, t \in F$ one has

$$
\begin{aligned}
D^{\prime}(\theta s) & =A^{-1} D(\theta(A s))=A^{-1}(d \theta \cdot A s)+(-1)^{p} \theta\left(A^{-1} \circ D \circ A\right) s \\
& =d \theta \cdot s+(-1)^{p} \theta \cdot D^{\prime} s,
\end{aligned}
$$

so that $D^{\prime}$ is a connection. Next,

$$
\begin{aligned}
\left\langle D^{\prime} s, t\right\rangle^{\prime}+\left\langle s, D^{\prime} t\right\rangle^{\prime} & =\left\langle A\left(A^{-1} D A\right) s, A t\right\rangle+\left\langle A s, A\left(A^{-1} D A\right) t\right\rangle \\
& =\langle D(A s), A t\rangle+\langle A s, D(A t)\rangle \\
& =d\langle A s, A t\rangle=d\langle s, t\rangle^{\prime}
\end{aligned}
$$

so that $D^{\prime}$ is metric with respect to $\langle,\rangle^{\prime}$. Finally, $K^{\prime} s=D^{\prime} D^{\prime} s=$ $\left(A^{-1} D D A\right) s=\left(A^{-1} K A\right) s$.

Lemma 2.2. For the metric connections $D, D^{\prime}$ of Lemma 2.1 it follows that $\mathrm{pf} K=\mathrm{pf}^{\prime} K^{\prime} \in \bigwedge^{2 n} \mathscr{E}$, where $\mathrm{pf}^{\prime}$ is defined with respect to $\langle,\rangle^{\prime}$.

Proof. Lemmas 1.4 and 2.1 give $*^{\prime} \circ \bigwedge^{n} K^{\prime}=\left(\bigwedge^{n} A^{-1} \circ * \circ \bigwedge^{n} A\right)$ 。 $\left(\bigwedge^{n} A^{-1} \circ \bigwedge^{n} K \circ \bigwedge^{n} A\right)=\left(\bigwedge^{n} A\right)^{-1 \circ}\left(* \circ \bigwedge^{n} K\right) \circ\left(\bigwedge^{n} A\right)$, hence

$$
\operatorname{tr} *^{\prime} \circ \bigwedge^{n} K^{\prime}=\operatorname{tr} * \circ \bigwedge^{n} K
$$

Now for any oriented $2 n$-plane bundle $\xi$ one imposes a riemannian metric on $\xi$ and computes pf $K \in \bigwedge^{2 n} \mathscr{E}$ for any connection $D$. The ChernWeil theorem guarantees that pf $K$ is closed and that the cohomology class [pf $K] \in H^{2 n}(M)$ is independent of $D$. (The Chern-Weil theorem can be presented entirely in terms of algebraic operations on $\Lambda^{*} \mathscr{E} \otimes \mathscr{F}$; see [6], e.g.) To show that [pf $K$ ] is also independent of the metric one 
observes that any two inner products on $F$ are related by $\langle e, f\rangle^{\prime}=\langle A e, A f\rangle$ for some invertible endomorphism $A: F \rightarrow F$; hence if $D$ is a metric connection with respect to $\langle$,$\rangle Lemma 2.1$ provides a metric connection $D^{\prime}$ with respect to $\langle,\rangle^{\prime}$ for which Lemma 2.2 gives $\left[\mathrm{pf}^{\prime} K^{\prime}\right]=[\mathrm{pf} K]$ as desired. Thus the cohomology class $(4 \pi)^{-n}[\mathrm{pf} K] \in H^{2 n}(M)$ depends only on $\xi$ itself; it will be denoted $e(\xi)$. The construction of pf $K$ guarantees naturality of $e(\xi)$, Proposition 1.7 provides the product formula $e(\xi \oplus \eta)=$ $e(\xi) e(\eta)$, and one verifies normalization as in [5], thus completing the axiomatic characterization of Euler classes.

The distinguishing feature of the preceding sketch is that one proves explicitly that $[\mathrm{pf} K]$ is independent of the riemannian metric on $\xi$. The same technique gives an instant proof of the Avez-Chern theorem (see [1], [2], and [3]), in which one imposes a pseudo-riemannian metric on $\xi$. We remark that a metric of type $(p, q)$ on $\xi$ induces an inner product $\langle,\rangle^{\prime}$ on $F$ with $\langle S, S\rangle^{\prime}=(-1)^{q}$ for any orientation $S \in \bigwedge^{2 n} F$; this alters none of the methods of this note. Here is the Avez-Chern theorem:

Proposition 2.3. Given a pseudo-riemannian metric on an oriented $2 n$-plane bundle $\xi$, let $\langle,\rangle^{\prime}$ be the induced inner product on $F$ and compute $\mathrm{pf}^{\prime}$ with respect to $\langle,\rangle^{\prime}$; then the Euler class $e(\xi)$ is represented by $(4 \pi)^{-n} \mathrm{pf}^{\prime} K^{\prime}$ for the curvature $K^{\prime}$ of any connection $D^{\prime}$ which is metric with respect to $\langle,\rangle^{\prime}$.

Proof. $\langle,\rangle^{\prime}$ induces an inner product $\langle,\rangle^{\prime}: F_{C} \times F_{C} \rightarrow R_{C}$ in the complexification $F_{C}$ of $F$. (Symmetric, not hermitian symmetric.) Let $\langle\rangle:, F_{C} \times F_{C} \rightarrow R_{C}$ be the inner product induced by any riemannian metric in $\xi$, so that $\langle e, f\rangle^{\prime}=\langle A e, A f\rangle$ for some invertible $A \in \operatorname{Hom}_{R_{C}}\left(F_{C}, F_{C}\right)$. If $*$ and $*^{\prime}$ are Hodge operators for $\langle$,$\rangle and$ $\langle,\rangle^{\prime}$ one has $*^{\prime}=\bigwedge^{n} A^{-1} \circ * \circ \bigwedge^{n} A$ as in Lemma 1.4 , and $A \circ D^{\prime} \circ A^{-1}$ is a metric connection $D$ with respect to $\langle$,$\rangle as in Lemma 2.1. Then$ pf $^{\prime} K^{\prime}=$ pf $K$ as in Lemma 2.2, which completes the proof.

We remark that Corollary 1.6 has not been used in this section. However, its sharpened form $(\mathrm{pf} K)^{2}=2^{2 n} \operatorname{det} K$ has a well-known application: $e(\xi)^{2}=p_{n}(\xi)$.

\section{REFERENCES}

1. A. Avez, Formule de Gauss-Bonnet-Chern en métrique de signature quelconque, C. R. Acad. Sci. Paris 255 (1962), 2049-2051; Rev. Un. Mat. Argentina 21 (1963), 191-197. MR 26 \#2993; 29 \#6452.

2. A. Borel, Sur une généralisation de la formule de Gauss-Bonnet, An. Acad. Brasil. Ci. 39 (1967), 31-37. MR 36 \#5866.

3. S. S. Chern, Pseudo-Riemannian geometry and the Gauss-Bonnet formula, An. Acad. Brasil. Ci. 35 (1963), 17-26. MR 27 \#5196. 
4. H. Flanders, On free exterior powers, Trans. Amer. Math. Soc. 145 (1969), 357367. MR 40 \#2662.

5. S. Kobayashi and K. Nomizu, Foundations of differential geometry, Vol. II, Interscience Tracts in Pure and Appl. Math., no. 15, Interscience, New York, 1969. MR 38 \#6501.

6. H. Osborn, Differential geometry in PL (to appear).

Department of Mathematics, University of IllinoIs, Urbana, Illinois 61801 